\title{
Binary Biometric Representation through Pairwise Polar Quantization
}

\author{
Chun Chen and Raymond Veldhuis \\ Signals and Systems Group, Electrical Engineering, University of Twente, \\ P.O. Box 217, 7500AE Enschede, The Netherlands \\ $\{c$. chen,r.n.j.veldhuis\}@utwente.nl
}

\begin{abstract}
Binary biometric representations have great significance for data compression and template protection. In this paper, we introduce pairwise polar quantization. Furthermore, aiming to optimize the discrimination between the genuine Hamming distance (GHD) and the imposter Hamming distance (IHD), we propose two feature pairing strategies: the long-short (LS) strategy for phase quantization, as well as the long-long (LL) strategy for magnitude quantization. Experimental results for the FRGC face database and the FVC2000 fingerprint database show that phase bits provide reasonably good performance, whereas magnitude bits obtain poor performance.
\end{abstract}

Keywords: Polar quantization, pairing, face, fingerprint recognition.

\section{Introduction}

Binary biometric representations have great significance for data compression and template protection [1. A common way to extract binary strings is by quantizing and coding the real-valued biometric templates. The binary string has to suffice the following two requirements:

1. To obtain a verification performance with low false acceptance rate (FAR) and false rejection rate (FRR), the binary strings are desired to be not only discriminative, but also robust to intra-class variation;

2. Considering template protection, the bits generated by the imposters should be independent and identically distributed, in order to maximize the efforts of guessing the genuine template.

To achieve the requirements, many work focus on designing one-dimensional quantizers, relying on the feature's statistical properties, e.g. the genuine user probability density function $(\mathrm{PDF}) p_{\mathrm{g}}$ and the background $\operatorname{PDF} p_{\mathrm{b}}[2],[3$, [4, 5], 6], 7]. Among them the fixed quantizer [5], 6] is global, constructed merely from the background PDF, whereas quantizers in [2, 3], 4], 7] are userspecific, constructed from both the genuine user PDF and the background PDF. Quantizers in [2], 3] and [4] have equal-width-intervals. Alternatively, quantizers in [5, 6], 7] have equal-probability-intervals. Furthermore, independent of 
the one-dimensional qantizers, the DROBA principle [8] was proposed to assign various numbers of quantization bits to every feature. In this paper we concentrate on the quantizer design. Although one-dimensional quantizers yield reasonably good performances, quantizing every feature independently ends up with inflexible quantization intervals, for instance, orthogonal boundaries in the two-dimensional feature space. Therefore, two-dimensional quantization might bring more flexible quantizer structures.

In this paper, we propose quantization in polar coordinates, including phase and magnitude. Unlike in audio and image coding 9], where polar quantization is directly applied on predetermined complex variables, quantizing biometric features allows freedom to compose the pairwise features. Furthermore, we define a measure of the discrimination between the quantized bits, by computing the inter-class and intra-class scatters between the genuine and the imposter Hamming distances. To optimize such discrimination, we develop the long-short (LS) and the long-long (LL) pairing strategies for phase and magnitude, respectively.

In Section 2 the polar quantization is introduced. In Section 3 we propose the long-short and the long-long pairing strategies, to optimize the discrimination between the genuine and the imposter Hamming distances. In Section 4, some experimental results are given for the FRGC face database and the FVC2000 fingerprint database, and conclusions are drawn in Section 5 .

\section{Polar Quantization}

Let $\mathbf{v}=\left\{v_{1}, v_{2}\right\}$ denote a two-dimensional feature vector. In polar coordinates, the phase $\theta$ and magnitude $r$ are:

$$
\begin{aligned}
& \theta=\operatorname{angle}\left(v_{1}, v_{2}\right), \\
& r=\sqrt{v_{1}^{2}+v_{2}^{2}},
\end{aligned}
$$

where $\theta$ is the counterclockwise angle from the $v_{1}$-axis, and $r$ is the radial distance from the origin. We assume that biometric features have circularly symmetric background PDF, feasible for polar quantization. A $n$-bit phase quantizer is then constructed as:

$$
\begin{aligned}
& \xi=\frac{2 \pi}{2^{n}}, \\
& Q_{\theta, i}=[(i-1) \xi \quad i \xi), i=1, \ldots 2^{n},
\end{aligned}
$$

where $Q_{\theta, i}$ represents the $i^{\text {th }}$ quantization interval within boundaries $[(i-1) \xi i \xi)$. When the background PDF is circularly symmetric, $\theta$ is uniformly distributed, leading to both equal- $\xi$-width and equal- $2^{-n}$-probability intervals.

A $n$-bit magnitude quantizer is constructed as:

$$
\begin{aligned}
B_{0} & =0 \\
B_{i} & =\arg _{B}\left[\int_{B_{i-1}}^{B} \int_{0}^{2 \pi} p_{\mathrm{b}}(\theta, r) d \theta d r=2^{-n}\right], i=1, \ldots 2^{n}, \\
Q_{\mathrm{r}, i} & =\left[\begin{array}{ll}
B_{i-1} & \left.B_{i}\right), i=1, \ldots 2^{n}
\end{array}\right.
\end{aligned}
$$


where $Q_{\mathrm{r}, i}$ represents the $i^{\text {th }}$ quantization interval within boundaries $\left[\begin{array}{ll}B_{i-1} & B_{i}\end{array}\right)$. Determining these intervals depends on the background PDF $p_{\mathrm{b}}$. The expression in (6) ensures equal- $2^{-n}$-probability intervals.

To summarize, both phase and magnitude quantization obtain equal background probability intervals. Thus, the imposters obtain independent and identically distributed bits.

\section{$3 \quad$ Feature Pairing}

\subsection{Hamming Distance Discriminant Analysis}

Often, binary biometric strings are matched via their Hamming distances. To design a verification system, a genuine Hamming distance (GHD) is computed when the query and the target share the same identity, otherwise an imposter Hamming distance (IHD) is computed. The decision is then made by applying a threshold $T$ to both distances. In this paper, we aim to optimize the discrimination between the GHD and IHD densities.

We begin by defining the discrimination between GHD and IHD. Suppose we have $P$ feature pairs. Each pair is quantized as a code $x_{i}, i=1, \ldots, P$, and subsequently concatenated into the binary string $X=x_{1}, \ldots, x_{P}$. Considering a genuine user $\omega$, with $\widehat{X}_{\omega}=\hat{x}_{\omega, 1} \ldots \hat{x}_{\omega, P}$ as the enrollment template. Let $d_{\omega, i}$ and $d_{\bar{\omega}, i}$ be the GHD and IHD for the $i^{\text {th }}$ feature pair, defined as:

$$
\begin{aligned}
& d_{\omega, i}=h\left(x_{i}, \hat{x}_{\omega, i}\right), x_{i} \in \text { user } \omega ; \\
& d_{\bar{\omega}, i}=h\left(x_{i}, \hat{x}_{\omega, i}\right), x_{i} \notin \operatorname{user} \omega,
\end{aligned}
$$

where function $h$ computes the Hamming distance between the two inputs. Then the GHD $\left(D_{\omega}\right)$ and the IHD $\left(D_{\bar{\omega}}\right)$ for the entire binary string are:

$$
\begin{aligned}
& D_{\omega}=\sum_{i=1}^{P} d_{\omega, i} ; \\
& D_{\bar{\omega}}=\sum_{i=1}^{P} d_{\bar{\omega}, i} .
\end{aligned}
$$

Furthermore, for the $i^{\text {th }}$ feature pair, we define the expectation of the GHD $\left(\bar{d}_{\omega, i}\right)$ and the IHD $\left(\bar{d}_{\bar{\omega}, i}\right)$ as:

$$
\begin{aligned}
\bar{d}_{\omega, i} & =\mathcal{E}\left[d_{\omega, i}\right], x_{i} \in \text { user } \omega ; \\
\bar{d}_{\bar{\omega}, i} & =\mathcal{E}\left[d_{\bar{\omega}, i}\right], x_{i} \notin \text { user } \omega .
\end{aligned}
$$

Thus, for the entire binary string, the expectation of the GHD $\left(\bar{D}_{\omega}\right)$ and the IHD $\left(\bar{D}_{\bar{\omega}}\right)$ become:

$$
\begin{aligned}
& \bar{D}_{\omega}=\sum_{i=1}^{P} \bar{d}_{\omega, i} ; \\
& \bar{D}_{\bar{\omega}}=\sum_{i=1}^{P} \bar{d}_{\bar{\omega}, i} .
\end{aligned}
$$


A measure of separation between GHD and IHD densities is their intra-class scatter and the inter-class scatter. Thus, we would like to minimize the intra-class scatter $S_{\text {intra, } \omega}$, defined as:

$$
S_{\text {intra }, \omega}=\mathcal{E}\left[\left(D_{\omega}-\bar{D}_{\omega}\right)^{2}\right]+\mathcal{E}\left[\left(D_{\bar{\omega}}-\bar{D}_{\bar{\omega}}\right)^{2}\right] .
$$

In the mean time, we want to maximize the inter-class scatter $S_{\text {inter, } \omega}$ :

$$
S_{\text {inter }, \omega}=\left(\bar{D}_{\bar{\omega}}-\bar{D}_{\omega}\right)^{2} .
$$

Substituting (10), (11), (14), (15) into (16) and (17), we have:

$$
\begin{aligned}
S_{\text {intra }, \omega} & =\mathcal{E}\left[\left[\sum_{i=1}^{P}\left(d_{\omega, i}-\bar{d}_{\omega, i}\right)\right]^{2}\right]+\mathcal{E}\left[\left[\sum_{i=1}^{P}\left(d_{\bar{\omega}, i}-\bar{d}_{\bar{\omega}, i}\right)\right]^{2}\right] ; \\
S_{\text {inter }, \omega} & =\left[\sum_{i=1}^{P}\left(\bar{d}_{\bar{\omega}, i}-\bar{d}_{\omega, i}\right)\right]^{2} .
\end{aligned}
$$

Assuming that the $P$ feature pairs are independent, $S_{\text {intra, } \omega}$ and $S_{\text {inter, } \omega}$ can be approximated as:

$$
\begin{aligned}
S_{\text {intra }, \omega} & =\sum_{i=1}^{P}\left[\mathcal{E}\left[\left(d_{\omega, i}-\bar{d}_{\omega, i}\right)^{2}\right]+\mathcal{E}\left[\left(d_{\bar{\omega}, i}-\bar{d}_{\bar{\omega}, i}\right)^{2}\right]\right]=\sum_{i=1}^{P} S_{\text {intra }, \omega, i} ; \\
S_{\text {inter }, \omega} & =\sum_{i=1}^{P}\left(\bar{d}_{\bar{\omega}, i}-\bar{d}_{\omega, i}\right)^{2}=\sum_{i=1}^{P} S_{\text {inter }, \omega, i} .
\end{aligned}
$$

Hence, the intra-/inter-class scatter for the entire binary string is simplified as the sum of the intra-/inter-class scatter over all the feature pairs. Usually biometric features are not presented in pairs, allowing the freedom to compose the pairwise features to optimize $S_{\text {intra, } \omega}$ and $S_{\text {inter, } \omega}$. Therefore, we formulate the problem as: for a genuine user $\omega$, to find a pairing configuration $\left\{C_{\omega, i}^{*}\right\}, i=$ $1, \ldots, P$, so that the discrimination between GHD and IHD is maximized:

$$
\begin{aligned}
\left\{C_{\omega, i}^{*}\right\} & =\arg \max _{\left\{C_{\omega, i}\right\}} \frac{S_{\text {inter }, \omega}}{S_{\text {intra }, \omega}}, \\
& =\arg \max _{\left\{C_{\omega, i}\right\}} \frac{\sum_{i=1}^{P} S_{\text {inter }, \omega, i}}{\sum_{i=1}^{P} S_{\text {intra }, \omega, i}} .
\end{aligned}
$$

\subsection{Pairing Solutions}

In order to optimize (21), we first have to analyze how the genuine user PDF relates to $S_{\mathrm{inter}, \omega, i}$ and $S_{\mathrm{intra}, \omega, i}$ in case of the polar quantization. Afterwards, we could decide a strategy to pair features with specific $p_{\mathrm{g}}$ to optimize $S_{\text {inter, } \omega, i}$ and $S_{\text {intra, } \omega, i}$. However, it is difficult to analytically find an expression for the relation, due to lack of samples and complex integral calculation in polar coordinates. Therefore, we employ an empirical method to simplify the relation of $S_{\text {inter, } \omega, i}$, $S_{\text {intra, },, i}$ and the genuine user PDF. We take two data sets: FRGC(version 1) [10] face database and FVC2000(DB2) fingerprint database [11. 
- FRGC: It contains 275 users with various numbers of images, taken under both controlled and uncontrolled conditions. A set of standard landmarks, i.e. eyes, nose and mouth, are used to align the faces. The raw measurements are the gray pixel values, leading to a total of 8762 elements.

- FVC2000: It contains 8 images of 110 different users. Images are aligned according to a standard core point position. The raw measurements contain two categories: the squared directional field in both $x$ and $y$ directions, and the Gabor response in 4 orientations $(0, \pi / 4, \pi / 2,3 \pi / 4)$. Determined by a regular grid of 16 by 16 points with spacing of 8 pixels, measurements are taken at 256 positions, leading to a total of 1536 elements [5].

We first apply PCA/LDA [12] to reduce both data sets into 50 features. Afterwards, for every genuine user, we randomly pair the features into 25 pairs. Following this pairing configuration, the entire data set, including the genuine user samples and the imposter samples are quantized via a 1-bit phase quantizer and a 1-bit magnitude quantizer, where the magnitude quantizer boundary is determined by a two-dimensional Gaussian density with zero mean and unit variance $p_{\mathrm{b}}(\mathbf{v})=N(\mathbf{v}, 0,1)$. The $S_{\text {inter }, \omega, i}$ and $S_{\text {intra }, \omega, i}$ for every feature pair is then computed based on the quantized bits. We repeat this process for all the genuine users in the data set. Eventually, we average $S_{\text {inter, } \omega, i}$ and $S_{\text {intra, } \omega, i}$ over all features as well as all genuine users, so that the averaged $\bar{S}_{\text {inter, } \omega, i}$ and $\bar{S}_{\text {intra, } \omega, i}$ are neither user nor feature biased. Intuitively, we speculate that the distance $r_{\omega, i}$, - distance between the feature pair mean and the origin - dominates the inter- and intra-class scatter. To analyze, in Fig. 1, we plot the value of $\bar{S}_{\text {inter, } \omega, i}, \bar{S}_{\text {intra, } \omega, i}$ as sorted by $r_{\omega, i}$, for both phase bits and magnitude bits. Both data sets reveal the same relations: Fig. 1(a) suggests that for phase quantization, when $r_{\omega, i}$ increases, the inter-class scatter increases and the intra-class scatter decreases; Fig. 1(b) suggests that for magnitude quantization, when the distance $\left|r_{\omega, i}-B\right|$ between the feature pair mean and the magnitude boundary

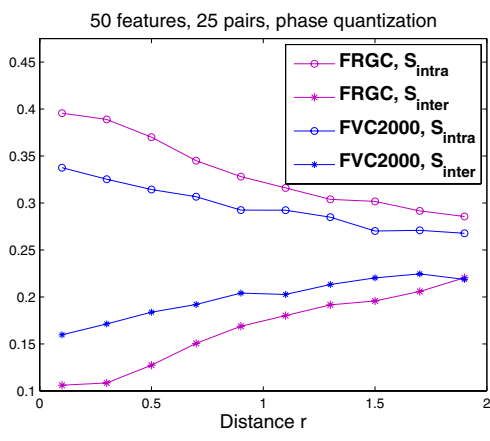

(a)

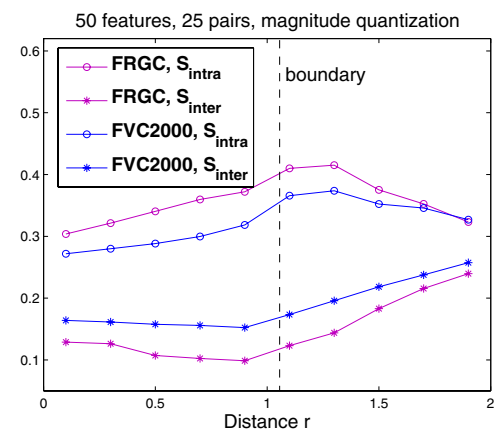

(b)

Fig. 1. The averaged $\bar{S}_{\text {inter }, \omega, i}$ and $\bar{S}_{\text {intra, }, i}$ for (a) phase and (b) magnitude, as sorted by $r_{\omega, i}$ 
increases, the inter-class scatter increases and the intra-class scatter decreases. Therefore, we simplify the problem (21) as:

$$
\begin{aligned}
& \left\{C_{\omega, i}^{*}\right\}=\arg \max _{\left\{C_{\omega, i}\right\}} \sum_{i=1}^{P} r_{\omega, i}, \text { for phase }, \\
& \left\{C_{\omega, i}^{*}\right\}=\arg \max _{\left\{C_{\omega, i}\right\}} \sum_{i=1}^{P}\left|r_{\omega, i}-B\right|, \text { for magnitude } .
\end{aligned}
$$

Optimizing the inter- and intra-class scatter is now simplified as optimizing the overall distance of the feature pairs. To solve (22) and (23), the straightforward way is to conduct a brute force search of all possible pairing configurations and pick the one with the maximum overall distance. Unfortunately, the computational complexity is too high. Therefore, we propose the following two heuristic pairing strategies: Given $2 P$ features, we first sort the mean of the $2 P$ features $\left\{\operatorname{abs}\left(\mu_{k}\right)\right\}, k=1, \ldots, 2 P$ from the smallest to the largest into a sequence of ordered feature indexes $\left\{I_{1}, I_{2}, \ldots, I_{2 P}\right\}$.

1. long-short (LS) strategy: The index of the $i^{\text {th }}$ feature pair is:

$$
C_{\omega, i}^{*}=\left(I_{i}, I_{2 P+1-i}\right), i=1, \ldots, P ;
$$

2. long-long (LL) strategy: The index of the $i^{\text {th }}$ feature pair is:

$$
C_{\omega, i}^{*}=\left(I_{2 \times i-1}, I_{2 \times i}\right), i=1, \ldots, P .
$$

Apparently, the long-short strategy selects features with a large mean and a small mean as a pair, keeping their distance $r_{\omega, i}$ large; thus, somehow maximizes the overall distances in (22) for phase quantization. Contrarily, long-long strategy selects features that either both have large means or both have small means, keeping their distance $r_{\omega, i}$ far away from the boundary; thus, maximizes the overall distances in (23) for magnitude quantization. The advantage of these two pairing strategies is that the computational complexity is low $O(P)$.

\section{Experiments}

We tested the polar quantization on the FRGC and the FVC2000 database, as described in Section 3.2. To first reduce the feature dimensionality, we applied $\mathrm{PCA} / \mathrm{LDA}[12$ on a training set, consisting of independent users from the enrollment and verification. The obtained transformation was then applied to both the enrollment and verification samples. In the enrollment, for every target user, assigned with Gray codes, the phase bits (phase+LS) were generated following the LS pairing strategy, while the magnitude bits (magnitude+LL) were generated following LL pairing strategy. By concatenating both the phase and the magnitude bits we obtained the total polar bits (polar+combined). The quantized codes, together with the pairing configuration $\left\{C_{\omega, i}^{*}\right\}$, was stored for each 
target user. During the verification, features of the query user were quantized according to the $\left\{C_{\omega, i}^{*}\right\}$ of the claimed identity, resulting in a query binary string. Eventually the verification performance was evaluated by a Hamming distance classifier. With, in total, $n$ samples per user $(n=8$ for FVC2000, and $n$ ranges from 6 to 48 for FRGC), the division of the data is indicated in Table 1

Table 1. Training, enrollment and verification data (number of users $\times$ number of samples per user) and the number of partitionings for FRGC and FVC2000

\begin{tabular}{|l|r|r|r|r|}
\hline & Training & Enrollment & Verification & Partitionings \\
\hline FRGC & $210 \times n$ & $65 \times 2 n / 3$ & $65 \times n / 3$ & 5 \\
FVC2000 & $80 \times n$ & $30 \times 3 n / 4$ & $30 \times n / 4$ & 20 \\
\hline
\end{tabular}

Since both the phase and the magnitude have fixed equal-probability-intervals, we compared their performances with the one-dimensional fixed quantizer (1D fixed) [5], 6], which has the same property. We first investigated the 1-bit quantization $\left(n_{\theta}=n_{\mathrm{r}}=n_{\mathrm{f}}=1\right)$ performances of phase+LS, magnitude+LL and polar+combined. The EER results for the FRGC and the FVC2000 at various feature dimensionalities are shown in Fig. 2. In general, the magnitude bits give poor performances, whereas the phase bits consistently yield good performances and outperform 1D fixed quantization. Furthermore, since the magnitude bits are so poor, combining both phase and magnitude bits, as seen with polar+combined, does not show good performance.

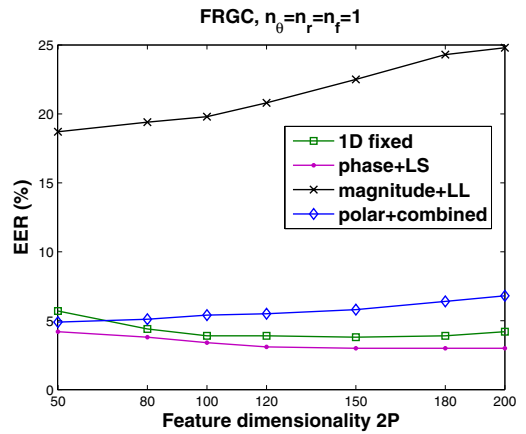

(a)

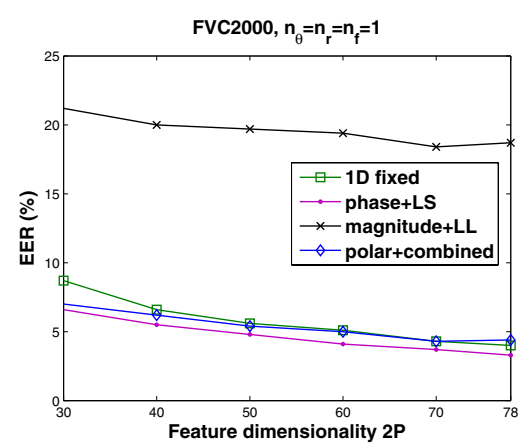

(b)

Fig. 2. The EER performances of phase+LS, magnitude+LL and polar+combined, as compared to the 1D fixed quantization, for (a) FRGC and (b) FVC2000

In Fig. 3 we further illustrate the GHD and IHD densities of the phase and the magnitude bits, at $P=60$ for FRGC, as compared to the $1 \mathrm{D}$ fixed quantization. We observe that for the three types of bits, the mean of their IHD densities are all around 0.5, demonstrating the equal-probability-intervals. The IHD density 


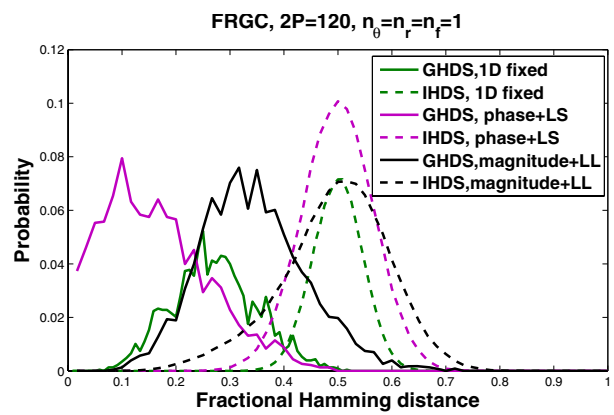

Fig. 3. The GHD and the IHD densities of phase+LS, magnitude+LL, and the $1 D$ fixed quantization, at $2 P=120$ for FRGC

Table 2. The inter-class and intra-class scatter of phase+LS, magnitude+LL and the 1D fixed, for (a) FRGC and (b) FVC2000

(a)

\begin{tabular}{|l|r|r|r|r|r|r|}
\hline FRGC & \multicolumn{3}{|c|}{$2 \mathrm{P}=50$} & \multicolumn{3}{|c|}{$2 \mathrm{P}=120$} \\
\cline { 2 - 7 } & 1D fixed & phase+LS & magnitude+LL & 1D fixed & phase+LS & magnitude+LL \\
\hline$S_{\text {inter }}\left(\times 10^{-2}\right)$ & 7.2 & 14.9 & 4.4 & 5.2 & 11.5 & 2.3 \\
$S_{\text {intra }}\left(\times 10^{-2}\right)$ & 1.3 & 2.0 & 2.7 & 0.8 & 1.4 & 2.0 \\
\hline
\end{tabular}

(b)

\begin{tabular}{|l|r|r|r|r|r|r|}
\hline FVC2000 & \multicolumn{3}{|c|}{$2 \mathrm{P}=50$} & \multicolumn{3}{|c|}{$2 \mathrm{P}=78$} \\
\cline { 2 - 7 } & 1D fixed & phase+LS & magnitude+LL & 1D fixed & phase+LS & magnitude+LL \\
\hline$S_{\text {inter }}\left(\times 10^{-2}\right)$ & 7.2 & 14.6 & 4.3 & 6.7 & 13.5 & 3.7 \\
$S_{\text {intra }}\left(\times 10^{-2}\right)$ & 1.2 & 2.1 & 2.8 & 1.0 & 1.6 & 2.2 \\
\hline
\end{tabular}

of the $1 \mathrm{D}$ fixed quantizer is relatively narrow, compared to those of the phase and magnitude. Additionally, the GHD density of the phase bits are located farther away from the IHD. Unfortunately, the GHD densities of the three types of bits are all quite wide.

The discrimination property explained above can be quantified as the interand intra-class scatter. Some examples computed on the fractional Hamming distances are given in Table 2. Consistent to what we observed in Fig. 3, for phase+LS, the $S_{\text {inter }}$ is on average two times the 1D fixed. In the mean time, its $S_{\text {intra }}$ does not increase much. For this reason, phase+LS yields better performance. On the other hand, magnitude+LL obtains smaller $S_{\text {inter }}$ and larger $S_{\text {intra }}$, leading to poor performance. Based on these analysis, we could conclude that even with carefully paired features, the magnitude does not provide discriminative bits for classification. Contrarily, the phase provides reasonably discriminative bits for classification.

In fact, the 1-bit phase quantizer, with boundary at $\pi$, quantizes only the feature that has a larger mean in the pairs, leaving the other feature discarded. Thus it also acts as a feature selection procedure. Now we test the phase quantizer 
with more quantization bits at $n_{\theta}=1,2,3,4$, compared to the $1 \mathrm{D}$ fixed quantizer at $n_{\mathrm{f}}=1,2$. Their EER results for the FRGC and the FVC2000 are shown in Fig. 4. Note that when $n_{\theta}=2$, in the two-dimensional feature space, the phase quantizer has the same orthogonal boundaries as the 1D fixed quantizer at $n_{\mathrm{f}}=1$, leading to the same performances. Results show that at a given feature dimensionality, phase bits at $n_{\theta}=1$ always give the best performances, while $n_{\theta}=2,3$ also yield reasonably good performances. Unfortunately, when $n_{\theta}=4$, the performances turn poor. Generally, compared to the 1D fixed quantizer, the phase quantizer gives better performances at a lower bit length.

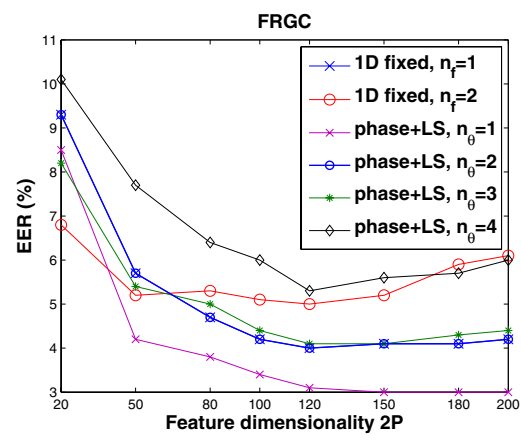

(a)

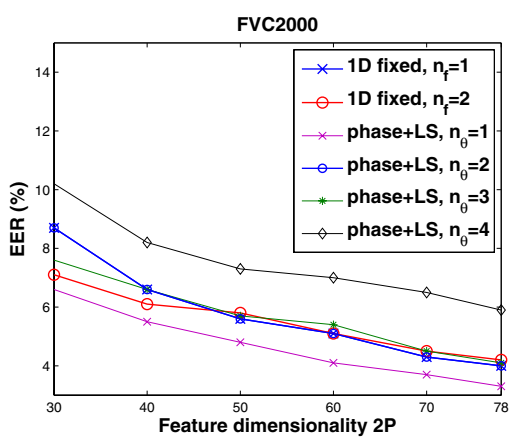

(b)

Fig. 4. The EER performances of phase+LS and 1D fixed quantization at various feature dimensionalities with various quantization bits, for (a) FRGC and (b) FVC2000

To summarize, although the magnitude $r_{\omega, i}$ itself does not provide discriminative bits, it in fact facilitates generating better phase bits. Additionally, the phase quantization has the following properties: (1) The LS pairing strategy is universal and simple, without modeling the specific genuine feature PDF; (2) The phase quantizer boundaries are not necessarily orthogonal, allowing correlations between the two features. Furthermore, the phase is uniformly distributed as long as the background PDF is circularly symmetric, which easily fits many feature modalities.

\section{Conclusions}

In this paper, we propose extracting binary biometric bits through polar quantization. In addition to the polar quantizer structure, quantizing features in pairs allows feature pair configuration. Therefore, we propose the long-long and the long-short pairing strategies to optimize the discrimination between the genuine Hamming distance and the imposter Hamming distance. Experimental results on the FVC2000 and the FRGC database show that magnitude yields poor classification performances, whereas phase provides reasonably good performances. 


\section{Acknowledgments}

This research is supported by the research program Sentinels (www.sentinels.nl). Sentinels is financed by Technology Foundation STW, Netherlands Organization for Scientific Research (NWO), and the Dutch Ministry of Economic Affairs.

\section{References}

1. Jain, A.K., Nandakumar, K., Nagar, A.: Biometric template security. EURASIP Journal on Advances in Signal Processing 8(2) (2008)

2. Vielhauer, C., Steinmetz, R., Mayerhofer, A.: Biometric hash based on statistical features of online signatures. In: Proc. 16th International Conference on Pattern Recognition (ICPR 2002), Quebec, Canada, vol. 1, pp. 123-126 (2002)

3. Hao, F., Wah, C.C.: Private key generation from on-line handwritten signatures. Information Management \& Computer Security 10(4), 159-164 (2002)

4. Chang, Y., Zhang, W., Chen, T.: Biometrics-based cryptographic key generation. In: Proc. IEEE International Conference on Multimedia and Expo. (ICME 2004), Taipei, Taiwan, vol. 3, pp. 2203-2206 (2004)

5. Tuyls, P., Akkermans, A.H.M., Kevenaar, T.A.M., Schrijen, G.J., Bazen, A.M., Veldhuis, R.N.J.: Practical biometric authentication with template protection. In: Kanade, T., Jain, A., Ratha, N.K. (eds.) AVBPA 2005. LNCS, vol. 3546, pp. 436446. Springer, Heidelberg (2005)

6. Kevenaar, T.A.M., Schrijen, G.J., van der Veen, M., Akkermans, A.H.M., Zuo, F.: Face recognition with renewable and privacy preserving binary templates. In: Proc. IEEE Workshop on Automatic Identification Advanced Technologies (AutoID 2005), NY, USA, pp. 21-26 (2005)

7. Chen, C., Veldhuis, R.N.J., Kevenaar, T.A.M., Akkermans, A.H.M.: Multi-bits biometric string generation based on the likelihood ratio. In: Proc. IEEE Conference on Biometrics: Theory, Applications and Systems (BTAS 2007) (2007)

8. Chen, C., Veldhuis, R.N.J., Kevenaar, T.A.M., Akkermans, A.H.M.: Biometric binary string generation with detection rate optimized bit allocation. In: IEEE Computer Society Conference on Computer Vision and Pattern Recognition Workshops (CVPR 2008) (2008)

9. Pobloth, H., Vafin, R., Kleijn, W.B.: Multivariate block polar quantization. IEEE Transactions on Communications 53(12), 2043-2053 (2005)

10. Phillips, P.J., Flynn, P.J., Scruggs, W.T., Bowyer, K.W., Chang, J., Hoffman, K., Marques, J., Min, J., Worek, W.J.: Overview of the face recognition grand challenge. In: Proc. IEEE Computer Society Conference on Computer Vision and Pattern Recognition (CVPR 2005), MD, USA, pp. 947-954 (2005)

11. Maio, D., Maltoni, D., Cappelli, R., Wayman, J.L., Jain, A.K.: FVC 2000: Fingerprint verification competition. IEEE Transactions on Pattern Analysis and Machine Intelligence 24(3), 402-412 (2002)

12. Veldhuis, R.N.J., Bazen, A., Kauffman, J., Hartel, P.: Biometric verification based on grip-pattern recognition. In: Proc. SPIE Security, Steganography, and Watermarking of Multimedia Contents VI (SSWMC 2004), CA, USA, vol. 5306, pp. 634-641 (2004) 